\title{
Glycomic profiling of glycoproteins
}

\author{
Jarkko Räbinä
}

Published online: 25 December 2012

(C) Springer Science+Business Media New York 2012

It has been known already since the 1950s that carbohydrates encode biological information, such as variation of blood group determinants. In the light of their importance, glycans have received relatively little attention and remained less studied than nucleic acids and proteins. However, with the increasing availability of improved tools designed to analyze protein-linked glycans in a sensitive and reproducible manner, the understanding of the roles of glycans in a variety of living systems and processes is rapidly rising. Many new glycan functions are now emerging from the studies of tissues and organs of humans and model systems, as well as in the area of microbial glycobiology.

Glycans are present on at least $50 \%$ of the proteins in the human proteome and nearly all of the secreted and cell surface proteins, playing an important role in almost every biological process and being involved in health and major diseases. Profiling of glycomic changes is increasingly important in searching biomarkers for diseases and the diagnostic potential of glycomic profiling for detecting cancer and various other disease states has been shown in numerous studies [1, 2]. Further, glycosylation is involved in many specific recognition events between microbial pathogens and their hosts, which are influenced to an important degree by the glycans and glycan-binding proteins that are expressed on the cell surfaces of either the pathogens or the host [3]. In addition to host-pathogen recognition, glycans also have functions in mediating symbiotic relationships with beneficial microbes [4].

An increasing number of glycoproteins are commonly utilized as biopharmaceutical products, including antibodies, glycoprotein hormones, and clotting factors. Seven of the current top ten biopharmaceuticals are glycosylated [5], which is likely to influence their biological efficacy. A striking

J. Räbinä $(\bowtie)$

Cell Surface Analytics Laboratory, Advanced Therapies

and Product Development, Finnish Red Cross Blood

Service, Kivihaantie 7,

00310 Helsinki, Finland

e-mail: jarkko.rabina@bts.redcross.fi example is $\mathrm{IgG}$, as glycans attached to the Fc portion are known to modulate its biological activity by affecting the interaction with both complement and various cellular Fc receptors $[6,7]$. The anti-inflammatory effect of plasmaderived intravenous $\mathrm{IgG}$ is mediated through sialylated $\mathrm{N}$ glycans, which represent a minor population of $\mathrm{N}$-glycans in the Fc portion [8]. In addition, optimal glycosylation is critical for both efficacy [9] and safety [10, 11] of therapeutic monoclonal antibodies, calling for high-throughput methods to profile glycosylation of biotechnologically produced IgG [12]. Similar needs for glycan profiling apply in analyzing cells aimed at therapy, which quite often mediate their function by cell surface glycan structures [13].

During recent years, a variety of new methods for sample preparation, analytics, and bioinformatics have emerged in the field of glycomics and become available to a wider group of researchers. In this special issue, entitled "Glycomic Profiling of Glycoproteins", we present some of the recent progress and development of novel analytical techniques in this important area leading to a more complete understanding of the functions of glycan moieties on glycoproteins.

M. Novotny and his colleagues describe in their comprehensive review many of the approaches and their limitations in conducting glycoproteomic studies. They summarize the analytical advances made during the past several years in the structural and quantitative determinations of glycoprotein glycans in complex biological mixtures. The needs for high-sensitivity glycan measurements from biological fluids and tissue extracts have been aided significantly by the use of miniaturized techniques both in sample preparation and analytical methods, leading to sensitivity gains amounting to several orders of magnitude.

J. Nilsson and colleagues focus in their review on presenting recent strategies specifically aimed at identifying the glycoproteome of cultured cells and biological samples, using targeted and global enrichment procedures and utilizing the high resolution power of nano-LC-MS/MS analyses. In particular they focus on targeted O-glycoproteomic analysis, both on the level of a single glycoprotein and of the 
global O-glycoproteome, covering thus an important aspect not dealt with in many other reviews. Various means of isolating and characterizing glycoproteins are presented that yield data both for the glycan structures and their attachment sites on the polypeptide sequence, such structural information being of fundamental importance for understanding the biological roles of complex glycosylations.

P. Rudd and coworkers provide an overview on glycoanalytical techniques as well as various fields of application with an emphasis on chromatographic techniques, used in profiling and defining the structures of $\mathrm{N}$-glycans. The new developments include the use of new chromatographic packing materials, a multiplexed labeling strategy, and a robotic platform for high-throughput analysis. State-of-theart glycan analysis technologies are required at all stages during the production of recombinant glycotherapeutics. Further, the analytical strategies developed by the group have been used for the discovery of biomarkers for disease, such as cancer, and the underlying mechanisms that result in glycosylation changes. High-throughput glycan profiling enables glycome/genome studies in large populations, allowing the integration of glycomics into other -omics platforms in a systems biology context.

M. Wuhrer's group has recently shown that antigen specific pathogenic antibodies differ in their glycosylation from the total serum pool of IgG in neonatal alloimmune thrombocytopenia [14]. Further, they have published many improvements to glycosylation analyses of biotechnologically produced therapeutic antibodies, which have become an important analytical challenge in the pharmaceutical industry. Here they discuss the question whether the transport of IgG to the fetus is biased by glycosylation. State-of-the-art methodology for Fc-glycosylation analysis at the glycopeptide level was applied. In contrast to earlier reports, the analysis revealed largely comparable Fc glycosylation for fetal and maternal IgG samples, suggesting that the materno-fetal IgG transport is not $\mathrm{Fc}$-glycosylation-selective in healthy pregnancies.

$\mathrm{H}$. Peltoniemi and colleagues disclose a glycan analysis software tool for the analysis of LC-MS/MS data to identify and profile glycan compositions. They report here the use of the glycan analysis software GlycanID in the glycan profiling of intravenous IgG and human fibroblast cell surface proteins. The latter LC-MS data revealed over 900 features, emphasizing the need for automated data analysis. The data handling workflow utilized existing proteomic software in combination with the new glycan specific tool, resembling thus the protocol used in many proteomics laboratories for the analysis of protein digests. This type of approach could allow laboratories to relatively easily perform glycomics analyses in addition to proteomics.

S. Nishimura and colleagues present an adaptation of their glycoblotting technique to the degradation products of hyaluronic acid. By utilizing hydrazide polymer beads oligosaccharides can be enriched by selective covalent capturing from a crude mixture via their reducing ends. As an advantage over previous methods, attachment to the beads provides rapid glycan sample preparation and enrichment, enabling automated high-throughput sample preparation. Their group has previously described the use of the technique for both N-glycan [15] and O-glycan [16] sample preparation. The present article communicates a glycoblotting approach for the characterization of hyaluronan fragments liberated from different sources of hyaluronan and discusses the functional roles of hyaluronan degradation products as signal molecules regulating cellular responses.

K. Khoo's group describes the identification of sulfated LacdiNAc epitope using both positive and negative ion MALDI-MS and ESI-MS/MS. The sulfated N- and Oglycans are problematic to be detected due to their negative charges and have often been neglected in glycan analytics. This report demonstrates that permethylated sulfated glycans produce stronger signals in the negative ionization mode using MALDI than in the positive mode. Permethylation neutralizes the charge contributed by the sialic acids, thus leaving the sulfated glycans as the only negatively charged glycans left, allowing optional fractionation to enrich sulfated glycans, and selective detection in negative ion mode. This phenomenon was used to detect sulfated LacdiNAc, an epitope identified on several pituitary glycohormones, with a well-defined role in mediating their clearance from blood circulation. The approach was demonstrated here with both thyroid stimulating hormone and ovarian cancer cell line and will potentially find application in the analysis of biologically significant sulfated glycans, leading possibly to identification of a wider occurrence of sulfated LacdiNAc epitope.

\section{References}

1. Alley Jr., W.R., Madera, M., Mechref, Y., Novotny, M.V.: Chipbased reversed-phase liquid chromatography-mass spectrometry of permethylated N-linked glycans: a potential methodology for cancer-biomarker discovery. Anal. Chem. 82, 5095-5106 (2010)

2. Arnold, J.N., Saldova, R., Galligan, M.C., Murphy, T.B., MimuraKimura, Y., Telford, J.E., Godwin, A.K., Rudd, P.M.: Novel glycan biomarkers for the detection of lung cancer. J. Proteome Res. 10, 1755-1764 (2011)

3. Imberty, A., Varrot, A.: Microbial recognition of human cell surface glycoconjugates. Curr. Opin. Struct. Biol. 18, 567-576 (2008)

4. Wacklin, P., Makivuokko, H., Alakulppi, N., Nikkila, J., Tenkanen, H., Rabina, J., Partanen, J., Aranko, K., Matto, J.: Secretor genotype (FUT2 gene) is strongly associated with the composition of Bifidobacteria in the human intestine. PLoS One 6, e20113 (2011)

5. Walsh, G.: Biopharmaceutical benchmarks 2010. Nat. Biotechnol. 28, 917-924 (2010)

6. Arnold, J.N., Wormald, M.R., Sim, R.B., Rudd, P.M., Dwek, R.A. The impact of glycosylation on the biological function and 
structure of human immunoglobulins. Annu. Rev. Immunol. 25, 21-50 (2007)

7. Raju, T.S.: Terminal sugars of Fc glycans influence antibody effector functions of IgGs. Curr. Opin. Immunol. 20, 471-478 (2008)

8. Kaneko, Y., Nimmerjahn, F., Ravetch, J.V.: Anti-inflammatory activity of immunoglobulin $\mathrm{G}$ resulting from Fc sialylation. Science 313, 670-673 (2006)

9. Jefferis, R.: Glycosylation as a strategy to improve antibody-based therapeutics. Nat. Rev. Drug Discov. 8, 226-234 (2009)

10. Bosques, C.J., Collins, B.E., Meador 3rd, J.W., Sarvaiya, H., Murphy, J.L., Dellorusso, G., Bulik, D.A., Hsu, I.H., Washburn, N., Sipsey, S.F., Myette, J.R., Raman, R., Shriver, Z., Sasisekharan, R., Venkataraman, G.: Chinese hamster ovary cells can produce galactose-alpha-1,3-galactose antigens on proteins. Nat. Biotechnol. 28, 1153-1156 (2010)

11. Ghaderi, D., Taylor, R.E., Padler-Karavani, V., Diaz, S., Varki, A.: Implications of the presence of N-glycolylneuraminic acid in recombinant therapeutic glycoproteins. Nat. Biotechnol. 28, 863-867 (2010)

12. Reusch, D., Haberger, M., Selman, M.H., Bulau, P., Deelder, A.M., Wuhrer, M., Engler, N.: High-throughput work flow for
IgG Fc-glycosylation analysis of biotechnological samples. Anal. Biochem. 432, 82-89 (2013)

13. Hirvonen, T., Suila, H., Kotovuori, A., Ritamo, I., Heiskanen, A., Sistonen, P., Anderson, H., Satomaa, T., Saarinen, J., Tiitinen, S., Rabina, J., Laitinen, S., Natunen, S., Valmu, L.: The i blood group antigen as a marker for umbilical cord blood-derived mesenchymal stem cells. Stem Cells Dev. 21, 455-464 (2012)

14. Wuhrer, M., Porcelijn, L., Kapur, R., Koeleman, C.A., Deelder, A., de Haas, M., Vidarsson, G.: Regulated glycosylation patterns of $\mathrm{IgG}$ during alloimmune responses against human platelet antigens. J. Proteome Res. 8, 450-456 (2009)

15. Amano, M., Nishimura, S.: Large-scale glycomics for discovering cancer-associated $\mathrm{N}$-glycans by integrating glycoblotting and mass spectrometry. Methods Enzymol. 478, 109-125 (2010)

16. Miura, Y., Kato, K., Takegawa, Y., Kurogochi, M., Furukawa, J., Shinohara, Y., Nagahori, N., Amano, M., Hinou, H., Nishimura, S.: Glycoblotting-assisted O-glycomics: ammonium carbamate allows for highly efficient o-glycan release from glycoproteins. Anal. Chem. 82, 10021-10029 (2010) 